Sādhanā, Vol. 19, Part 2, April 1994, pp. 271-287. (C) Printed in India.

\title{
Solid finite elements through three decades
}

\section{N VENKATESH and U SHRINIVASA*}

Department of Mechanical Engineering, Indian Institute of Science, Bangalore 560012, India

E-mail : udipi@mecheng.iisc.ernet.in

Abstract. Conventionally, solid finite elements have been looked upon as just generalizations of two-dimensional finite elements. In this article we trace their development starting from the days of their inception. Keeping in tune with our perceptions on developing finite elements, without taking recourse to any extra variational techniques, we discuss a few of the techniques which have been applied to solid finite elements. Finally we critically examine our own work on formulating solid finite elements based on the solutions to the Navier equations.

Keywords. Solid finite elements; extra-variational techniques; interpolation functions; convergence criteria; Papcovitch-Neuber solution; Navier equation.

\section{Introduction}

Development of the finite element method (FEM) as an analysis tool for continuum problems coincided with the advent of powerful digital computers. Using this method it is possible to establish and solve equations pertaining to complex systems in a very simple manner. It is precisely because of these reasons that today FEM has come to stay as a powerful tool in engineering analysis and design encompassing many diverse fields including structural mechanics, fluid mechanics, solid mechanics, electromagnetism etc. In fact it has emerged as a very popular analysis tool in interdisciplinary problems. The popularity of the method can also be attributed to the ease with which complex domains can be handled, requiring no additional techniques.

As in the case of all original development it is difficult to pinpoint exactly when FEM was discovered. It could be attributed to three separate groups - mathematicians (Courant 1943; Collatz 1950; Courant \& Hilbert 1953), physicists (Synge 1957) and engineers (Turner et al 1956). Very good surveys of the origins of FEM exist and are today commonly found in introductory chapters in many textbooks (Zienkiewicz 1973; Yang 1986). As is often the case with diverse groups working far removed from one another, emphasis on the various aspects of the study has been different for both engineers and mathematicians. Engineers were not deterred by the lack of an elegant mathematical theory, while mathematicians concerned themselves with those aspects of the problem which interested them more, like convergence. 
Today, FEM has been put on a relatively more sound mathematical footing. Extensive literature exists on its mathematical foundations and many aspects can be found elegantly represented in books (Ciarlet 1978; Oden 1983). FEM (as used by both mathematicians and engineers) involves a series of approximations. While it has generally been accepted that any procedure of discretization will involve a series of approximations, the approximations of FEM are more far reaching than others. For example, the breaking up of an elastic continuum itself is an approximation, for these elements are joined together only at the nodes.

Amongst the many other approximations made by the method, the most crucial one appears to be that of assuming that the displacements within the element can be expressed as a linear function of the nodal point displacements. This seems to have an overriding impact on the formulation of successful finite elements. We call this functional relationship from now on as the "interpolation function". This relationship is also referred to by various names in literature like shape functions, basis functions etc.

The choice of the geometry of the element and the interpolation function leaves a great deal of scope to the ingenuity and skill of the engineer designing finite elements. Solutions obtained would obviously depend a great deal on the exercise of this skill. FEM is normally implemented as the minimization of a functional. For example the popular displacement based approach, uses the principle of minimization of the total potential. The solutions obtained on minimization of the functional would then be obviously constrained by the choice of the assumed displacement field. Various types of interpolation functions are used in the literature. There have also been many methods and functionals used for formulation of finite elements. It is beyond the scope of this article to examine all of them. We shall therefore restrict ourselves to examining the methods and interpolation functions used in the formulation of threedimensional finite elements for elastostatics.

\section{3-Dimensional element formulations and interpolation functions}

Conventionally finite elements are formulated by strictly adhering to three cardinal principles, known as the "convergence criteria".

\subsection{The convergence criteria}

The assumed interpolation functions limit the infinite degrees of the system to a function with finite degrees of freedom. Therefore the minimum of the functional will not represent the correct equilibrium configuration as it would largely depend on number of degrees of freedom chosen. This will be true in spite of the fineness of the finite element mesh and the number of subdivisions used. Therefore, conventionally, in order to ensure convergence to the correct solution, certain criteria are required to be satisfied by the interpolation functions. They are the following (Zienkiewicz \& Taylor 1989).

(1) "The displacement function chosen should be such that it does not permit straining of an element to occur when the nodal displacements are caused by a rigid body displacement."

(2) "The displacement function has to be of such a form that if nodal displacements 
are compatible with a constant strain condition such constant strain will in fact be obtained."

(3) "The displacement functions should be so chosen that the strains at the interface between elements are finite."

Of course all the three criteria are needed to be satisfied only in the limit when the element sizes shrink to zero. Earlier they (or slightly modified versions of them) went by the names commonly referred to as continuity, compatibility, and a third criterion of completeness. Mathematical statements encompassing them exist in the name of "functional completeness" (de Arrantes Oliveira 1968; Strang \& Fix 1973). Unfortunately they do not speak about the rates of convergence, except recognizing that in the limit as element sizes go to zero (thereby calling for repeated mesh refinement), solutions converge. In fact it can been shown that all elements which have been formulated by rigorously adhering to the above criteria will converge (Strang \& Fix 1973). However, these criteria could not explain the phenomenon of locking and the resulting loss in convergence.

\subsection{Solid elements satisfying the convergence criteria}

We first consider solid elements developed by adhering to the above convergence criteria.

2.2a Tetrahedral elements: The first formulation of a simple tetrahedral element was by Gallagher $e t$ al in 1962 and was used in the stress analysis of heated complex shapes. Early elaborations of tetrahedral elements were by Melosh (1963), Argyris (1965) and Clough (1969). One of the early extensive numerical studies is due to Rashid (1969, 1970).

In these studies (and future elements to be discussed) tetrahedral elements were formulated in terms of volume coordinates similar to the triangular elements which were formulated in area coordinates, and were simple generalizations of the triangular elements. These elements were numerically integrated to obtain the element matrices.

As can be easily deduced the first of the tetrahedral elements (Gallagher et al 1962) was a $C^{0}$ continuous, 4-node, 12-degree-of-freedom (d.o.f.) constant strain tetrahedron, with linear shape functions along the three orthogonal cartesian directions.

Clough (1969) used a $C^{0}, 10$-node, 30 d.o.f. linear strain tetrahedron by adding midside nodes. This tetrahedron used complete quadratic polynomials in the three directions. Other higher order elements have also been formulated. Argyris et al (1968b) obtained a $C^{0}, 20$-node, 60 d.o.f. quadratic strain tetrahedron by adding nodes at the one- and two-third points of each of the six sides of the tetrahedron. The interpolation function was a complete twenty-term cubic polynomial in volume coordinates. Rashid et al (1969) used a $C^{0}, 16$-node, 48 d.o.f. tetrahedron by omitting the centroidal face nodes. Argyris et al (1968c) proposed the TEA8 element with 8 nodes and 60 d.o.f. This element has four centroidal nodes in addition to the four vertex nodes. Each vertex node has the following degrees of freedom: $u, \partial u / \partial x, \partial u / \partial y, \partial u / \partial z$ and similar d.o.f. in the $v$ and $w$ directions. Hughes \& Allik (1969) and Fjeld (1969) have formulated and used a 4-node, 48 d.o.f. tetrahedron by using the four vertex nodes and with d.o.f. of $u, v, w$ and their derivatives in the $x, y$ and $z$ directions at each node. Being a higher order element with derivative degrees of freedom, this element required higher order continuity, and as could be expected "this is the most advantageous tetrahedron introduced" (Yang 1986) till its date of publication. 
Comparative studies on the above tetrahedral elements abound (in a partial sense upto their dates of publication) and the interested reader is referred to the work of Fjeld (1969). Initial work regarding the development of tetrahedral elements were hampered due to the manual input of data required. With the advent of high speed computer graphics many algorithms can be found (Pammer \& Szabo 1981; Nguyen 1982) which aid the user in developing the data for the analysis.

2.2b Rectangular hexahedral elements based on the convergence criteria: Initial work on conforming hexahedral elements tended to be restricted to rectangular ones. This could be attributed to two reasons. (a) The generalized coordinate approach could yield geometries which were not invertible and (b) since the faces and sides of the rectangular elements are orthogonal to one another, such elements can be formulated using non-dimensional local coordinate systems. A number of such elements have been formulated. Amongst the first of these was a $C^{0}, 8$-node, 24 d.o.f. linear displacement rectangular hexahedron (Melosh 1963; Clough 1969). The element used "trilinear" displacement interpolation functions in the three orthogonal directions. The stiffness matrix was explicitly given by Melosh (1963). The addition of one node to the midpoints of each of the 12 sides of the hexahedron gives a $C^{0}, 20$-node, 60 d.o.f. quadratic displacement hexahedron. Just like the trilinear element used incomplete cubic polynomials, this element used incomplete quartic ones. This element was first enunciated by Clough (1969) and highlighted by Rigby \& McNeice (1972) and Pawsey \& Clough (1971). The addition of two nodes, at the $1 / 3$ and $2 / 3$ points of the edges yields a $C^{0}, 32$-node, 96 d.o.f. cubic displacement rectangular hexahedron, which uses incomplete quintic polynomials, see Ergatoudis et al (1968). Other higher order elements have also been formulated. The addition of four facial nodes on each of the 6 faces of the hexahedron in addition to the 32-node hexahedron above and the addition of 8 interior nodal points yields a 54-node, 192 d.o.f. $C^{0}$ hexahedral element, first used by Argyris \& Fried (1968). Here the interpolation function can be obtained by taking the product of 3 cubic polynomials in 3 directions. This of course leads to an incomplete higher-order field (such elements are known as Lagrangian elements). Another commonly used rectangular Lagrangian element is the one obtained by taking the product of quadratic polynomials in three orthogonal directions to obtain a 27 -node, 54 d.o.f. hexahedron with a centroidal node. Since no loads could be applied on the centroidal node, the d.o.f. corresponding to this node are statically condensed.

The Lagrangian elements have a disadvantage in that the interpolation functions require the use of unusually large degrees of the polynomial for interpolation.

The use of first derivative degrees of freedom (along the 3 directions $x, y$ and $z$ ) yields a 8 -node brick with 12 d.o.f. at each node ( 96 d.o.f). For such an element the displacement functions could be assumed in the form of Hermitian polynomials as in Argyris et al (1968b) or an incomplete fifth order field as used by Zienkiewicz et al (1970).

On account of the fact that 3-dimensional elements require large matrices to be stored and enormous computational effort, numerical experiments concerning these solid elements have by and large been "spotty" (Yang 1986) as compared to their 2-dimensional counterparts. But it is obvious from the above discussion that a large number of elements of both tetrahedral and hexahedral geometries can be formulated. Some numerical comparisons do exist in literature. The interested reader is referred to studies by Melosh (1963), Rashid (1970), Hughes \& Allik (1969), Fjeld (1969), 
Chacour (1972) and Ferguson \& Clark (1979). For a brief idea (in tabular form) of the relative performance of each element (accuracy/unit computational time) the reader is referred to Yang (1986).

One other hexahedral element which has been formulated, though not frequently used is a fourteen-noded brick obtained by the use of 6 facial nodes in addition to the 8 vertex nodes. This element suffers from the drawback that it is very difficult to obtain a frame invariant element. Irons \& Ahmad (1979) have noted that even though the element was very promising as it would produce a small bandwidth/frontwidth for analysis "there is something wrong with this element". Kidger (1990) and Smith \& Kidger (1992) using averaging along three directions have produced 14-node hexahedral elements and obtained success with some problems.

2.2c Other elements: Finite elements of shapes other than tetrahedra and hexahedra have also been formulated. Some of these are wedge-shaped and pentahedral elements. For example, in the wedge-shaped (triangular prisms) the interpolation functions can be obtained as a product of the Lagrange approach and the serendipity approach. See Zienkiewicz (1977) for the interpolation functions used in formulating the 6-node18 d.o.f., 15-node-45 d.o.f. and 26-node-78 d.o.f. triangular prism elements. Many forms of pentahedral elements also exist. For one of these elements the reader is referred to Haggenmacher (1993).

\subsection{Solid elements based on hierarchic interpolation functions}

So far in all the elements mentioned above, we find that we need to increase the number of nodes when we want to increase the order of the interpolating polynomial or alternately use elements with derivative degrees of freedom. Yet another means of generating interpolating functions for elements is to use hierachic approximations. In this method one needs to associate the monomial term in each interpolating polynomial with just a parameter and not to one with an obvious physical meaning. The only constraint associated with these hierachic functions is that they need to have zero values at the end of the range - in this case the vertices or nodal points along the edge under consideration. Using these polynomials one can arrive at a variety of interpolation functions for elements of different geometries. In fact it is possible to obtain a general form for interpolation functions, as demonstrated by Peano (1976). For 3-dimensional elements "a simple identification of the hierarchic parameters on the interfaces will automatically ensure $C^{0}$ continuity of the approximation" (Zienkiewicz \& Taylor 1989). In order to obtain optimal forms of hierarchical functions - those that result in a diagonal equations system, it can be shown that such interpolations can be obtained using orthogonal polynomials, for example Legendre polynomials. The products of these interpolation functions in three directions can yield interpolation functions useful for 3-dimensional elements (Zienkiewicz et al 1983).

\subsection{Isoparametric elements}

It is obvious from the above discussion, that it is possible to generate a large number of solid finite elements very easily. Despite the ease of formulation, all the above mentioned elements suffer from one particularly severe drawback - their poor curve fitting ability. This ability is severely tested when modelling real life problems with complex curved geometries. 
In order to overcome this drawback isoparametric elements are used. The method allows for the use of a nonlinear transform, mapping of a given finite element geometry to a "parent" one. "The interpolation of the element coordinates and the element displacements using the same interpolation functions, which are defined in the natural coordinate system, is the basis of the isoparametric element formulation" (Bathe \& Wilson 1976). In addition another particular drawback of the generalized coordinate method is also overcome, namely, the excessive care which is to be exercised in order to express the generalized coordinates in terms of nodal point displacements involving the inversion of a matrix (which for certain geometries could be singular). Also it overcomes the increased computational effort required in the generalized coordinate approach to transform element matrices from the element (local) coordinate system to a global one - this is more easily done in the case of isoparametric elements. These advantages are achieved by the direct use of interpolation functions used for the displacement. Isoparametric elements are today very popular. The first isoparametric element was developed by Taig (Robinson 1985) in 1958, but the first work was published by Irons (1966). Since then many publications comparing one set of isoparametric elements with another and highlighting the advantages of these with respect to the generalized coordinate elements have appeared (Ergatoudis et al 1968; Clough 1969; Zienkiewicz et al 1969; Ahmad et al 1970). The interpolation functions used in the formulation were similar to those described in the previous section, except that interpolation functions for the displacement field were directly used.

The basic problems of using isoparametric elements (as in non-isoparametric formulations) are two fold (Zienkiewicz et al 1971; Pawsey \& Clough 1971; Wilson et al 1973). "Firstly excessive shear strain energy is stored in these elements and, secondly, as the element becomes "thin" (for example, very large $l / d$ ratios in the case of analysis of plates and shells) the stiffness coefficients corresponding to the transverse degrees of freedom are larger than those of longitudinal displacements, which results in numerical ill-conditioning" (Bathe \& Wilson 1976).

\section{Extra-variational techniques}

Most of the elements mentioned in the above sections were derived by adhering strictly to the convergence criteria (except the ones using hierarchic interpolations). The behaviour of these elements in situations - such as bending or near incompressiblity (especially the lower-order elements) left a lot to be desired. These problems, depending on the type being solved and the element under consideration, are known by various names. Three-dimensional elements are known to suffer from delayed compressibility locking respectively. Here, the term "locking" will be used to "denote an indefinite decay of accuracy in displacement recovery" (Naganarayana 1991). Locking is prevalent in other structural problems also, like shear locking in flat plates/shells (see Bathe \& Dvorkin (1985) and Donea \& Lamain (1987)) and membrane locking in curved beam and shell structures (Stolarski \& Belytschko 1981; Prathap 1985). Other common problems encountered are "violent stress oscillations" (Prathap 1992) and "delayed convergence" (Naganarayana 1991). It will not be wrong to suggest that the various new elements which are formulated lately address themselves to tackling these problems. In fact there does not appear to be any solid element which 
is totally divorced from these problems. Since the early days of the finite element method, the development of such elements has been the source of both a challenge as well as a motivation for element developers.

Many techniques do exist in literature to get around these problems rather than attempt to solve them. (Consistency (Prathap 1992) is an attempt at solving the problem, though not fully successful for solid elements.) These techniques have been labelled variously as "tricks" (Prathap 1992) by some or "variational crimes" (Strang 1972) by some others, for they do not strictly adhere to the exact rules laid down by the variational principles on which the mathematical model is based. Many of these techniques can be categorized as "ad-hoc" (Naganarayana 1991), for their success in some problems does not necessarily imply the same when extrapolated to other problems. The list of these techniques is very large and continues to grow even today. "The task of developing good finite elements never seems to be finished. Designers return, again and again, to the same basic configuration of nodes and find some way to eke out an improvement" (MacNeal 1992).

It would be a Herculean task to list the entire set of these techniques individually. We list below only some of the above mentioned techniques, representing a good sample of select procedures which over the years have come to stay - these techniques have been regarded as the "milestones" (MacNeal 1992) of progress in the FEM.

(1) Reduced/selective integration: This is among the first of the so called "variational crimes" discovered. In this method the strain energy is not integrated exactly. For example, the 8-node solid element is integrated using a $2 \times 2 \times 2$ Gauss rule and the 20 node brick by a $3 \times 3 \times 3$ Gauss rule. The 27 node Lagrangian element uses a $3 \times 3 \times 3$ order rule. It is well-known that an $n$ point rule in one dimension can be used to integrate a polynomial of order $2 n+1$ exactly (Stroud 1971; Conte \& de Boor 1980 ). So a $3 \times 3 \times 3$ rule is required to integrate the cubic interpolation function in the 8-node element exactly. But it has been observed that in practice the reduced integrated element converges faster to the exact solution. This technique which is widely used in the FEM is called reduced integration. These rules need to be used with care. A very low order rule can lead to mechanisms (Brassioulis 1989), while the use of a very high order one leads to delayed convergence. One common type of mechanism encountered during reduced integration is the presence of hour glass modes. Various methods have been proposed in order to control these modes for 8 noded hexahedral solid elements (Flaganan \& Belytschko 1981; Schulz 1985) and for 20-noded solid elements (Kelen 1989). Such rules have proved their mettle in many problems, particularly related to plate and shell flexure (Pawsey \& Clough 1971; Zienkiewicz et al 1971) and also when solid elements are degenerated into flat plate and curved shell elements. Explicit integration for such degenerated elements have led to difficulties, which require the use of special techniques (Vlachoutsis 1990). The success of this techniques has led to efforts to include the experience gained into updating finite element codes (Case \& Vandegrift 1986; Case et al 1986). Many explanations have been sought for the success. Some are heuristic. Others have been based on more scientific arguments. Prathap and co-workers (1992) have looked upon this technique as a method to obtain "field consistency". Zienkiewicz \& Taylor (1989) attribute the success to the fact that the Gauss points being used in reduced integration are exactly the optimal points for stress recovery. "However, the main reason for success does not lie here but is associated with the fact that it provides the necessary singularity of the constraint part of the matrix, which avoids locking" (Zienkiewicz \& 
Taylor 1989). It should also be noted here that for mapped elements (like the isoparametric ones) full integration will not exactly integrate a deformed element (Cook et al 1989).

(2) Addition of bubble modes (Wilson et al 1973; Wilson 1973): This technique involves the addition of certain degrees of freedom not associated with any node (like the hierarchic functions mentioned previously). This brought into use, incompatible elements, where the displacement fields are not continuous across element boundaries. The variables associated with the nodeless degrees of freedom are later condensed out using a static condensation procedure.

In the case of the 8-node brick element this technique when used in tandem with reduced integration, gives very good results and has found its way into many commercial finite element programs. Unfortunately, the use of this technique requires experience. While it is possible that certain polynomial terms can be associated with the element whose "shape functions" are known, no clues are available in literature to guide the novice as to which functions are to be or not to be chosen. The success of the technique appears to lie in the fact that the functions which are chosen are the exact ones required to remove only certain types of locking. For example, in the case of the 8-node brick element, the incompatible modes chosen alleviate parasitic shear stored during bending. It also alleviates locking near incompressible limits.

(3) Using unequal order interpolation: This is one of the simpler techniques in use, espcially in the case of 1- and 2-dimensional elements. For example, Tessler \& Dong (1981) have formulated one such Timoshenko beam element. Here the order of the interpolation function used for the rotational degrees of freedom is one less than that of the translational ones. Its success could be attributed to the fact that the terms dropped from the interpolation functions for the rotational degrees of freedom are exactly the ones which if present will cause locking. Unequal order interpolation has been used in the formulation of many solid finite elements, like transitional elements and solid elements in the analysis of plates (16-node hexahedral elements).

(4) Assumed strain methods (MacNeal 1982; Olesan 1983): This techniques involves the use of computation of interpolation functions (of lower order) and smoothing them in some least square sense. It has been felt that this procedure is equivalent to that of reduced integration (Prathap 1992), but it has an added advantage in that the procedure can be used to obtain interpolation functions, while in the case of reduced integration, the points of reduced integration may not exist.

(5) Residual energy balancing (Fried 1974, 1975; Cook 1977): In this technique certain constraints contributing to locking are identified. These constraints are then artificially removed by the use of a constant, which the designer of the finite element sets to an arbitrarily small value. The point to be noted here is that the arbitrary constant to be used is problem dependent and it appears to be difficult to choose one value for a set of elements and problems. The constant is also mesh dependent, thereby compounding to the confusion. Stresses predicted by this method are "very unreliable" (Prathap 1992) and grossly dependent on the value of the scaling constant chosen. This technique, it is felt, is extrapolatable to solid finite elements and hence must be used with care.

(6) Reduced interaction (Prathap 1992): In this case the field variable that causes locking is identified and replaced by a lower order interpolation field.

(7) Elements based on variational principles other than the principle of minimum total potential: These will be dealt with in a later section. 
There are a number of other ingenious techniques used to obtain better elements. Amongst the others are synthesis using Fourier components (Park 1984), the use of trigonometric interpolation functions (Heppler \& Hansen 1987), use of shear constraints (Crisfield 1984) etc. The list appears to grow even today, as newer techniques are developed to obtain elements which would predict accurate solutions (for both stresses and displacements). Obviously, the primary reason for the development of these elements could be attributed to the lack of success with those previously developed. Even the techniques mentioned above do not appear to provide satisfactory explanations for their success. While, sometimes they work well is one context, they do not do so in others. This has led to a compounding of the already prevailing confusion.

\section{Some other types of interpolation functions}

In addition to the interpolation functions mentioned above, various other types are still being developed. The hybrid-stress elements (see $\S 5$ below) which uses independent interpolations for stresses and displacements has been viewed as just another extravariational technique by some authors (Prathap 1992; Andelfinger \& Ramm 1993). Some authors have looked at the use of "rational" interpolation functions (Wachspress 1975) for formulating a hexahedral element (Wait 1971) and its applications (Yu 1990). Here interpolation functions which can be defined as the ratio of two polynomials are used. De Freitas \& Castro (1992) have proposed the use of digital interpolation functions (based on a class of functions known as the Walsh functions) and can be viewed as a method similar to the ones using trigonometric functions above. A new class of interpolation functions called "physical shape functions" have also been presented (Gilewski \& Gomulinski 1990), where physical parameters and material parameters are interpolated in addition to displacements. Bergan \& Nygard (1984) propose a "free formulation" where elements can be formulated by splitting up the displacement field into one with lower order fields and another with higher order ones. Even though it appears that these techniques have not yet been used in the design and development of solid finite elements, they can, in some sense, be described as generalized methods, and we feel they are extrapolatable to solid elements.

\section{The newer 8-node brick elements}

It has been seen that it is the lower-order hexahedral elements (in the case of twodimensional elements it is the quadrilateral ones) which suffer from locking due to parasitic shear. Therefore it is not surprising that recent developments of solid finite elements have addressed themselves to this problem. In this section we look at some "new" formulations of the 8-node brick element.

\subsection{Displacement-based elements}

We first look at the displacement-based elements (like all formulations discussed above). A number of such elements have been formulated. Wilson (1973) first proposed an incompatible element, with additional bubble modes in order to incorporate a method which ensured that the 8-node brick does not lock under bending situations. 
The element he proposed did not pass the patch test. Taylor et al (1976) by imposing a set of constraints which ensured that the element satisfies the patch test a priori, were able to arrive at an element which has remained popular to this day. Bretl \& Cook (1979) have proposed an 8-node element, in which the stiffness matrix corresponding to the lower-order strains and those of the higher-order ones have been separated out. Depending on the choice of the problem, the corresponding higher-order strains are used. The stiffness matrices of the lower-order fields use a stress-based approach different from the higher-order ones. Yunus et al (1991) have proposed an 8-node element with rotational degrees of freedom. This element which had three additional modes to overcome Poisson ratio locking, is prone to experience zero energy modes which are alleviated using additional procedures. (The method is similarly extended to the development of tetrahedral elements with rotational degrees of freedom (Pawlak et al 1991). All the displacement-based elements discussed so far predict poor stresses under many loading conditions. Wilson \& Ibrahimbegovic (1990) using a least squares approximation to extract stresses were able to improve the stress prediction of the 8-node brick element with incompatible modes-stress predictions were identical to that predicted by stress-based elements. In this way they were able to separate the procedures for computing the stresses and for displacements.

\subsection{Elements based on other variational principles}

The discussions above highlight the difficulties encountered by designers in the formulation of displacement based elements. Over the years elements based on other variational principles have been developed.

Prathap and co-workers (1992) have attempted to obtain explanations for the problem of locking and to formulate elements which do not take recourse to any of the extra-variational techniques. They use the "field consistency paradigm" and the "functional reconstitution technique" to obtain elements which they show do not lock. This method appears to call for a variation of the strain fields independent of the displacements and is based on the more general Hellinger-Reissner and the $\mathrm{Hu}$-Washizu principles (Washizu 1968). Moreover the solid elements which have been formulated using this technique suffer from one severe drawback in that the 8-node brick formulated (Chandra \& Prathap 1989) is very sensitive to geometric distortions and predicts poor stress values (Dong \& de Freitas 1992). This has been attributed to the difficulties encountered in finding a "technique of consistent mapping for covariant finite element formulation in 3-D applications..." (Naganarayana 1991). In some cases (Naganarayana \& Prathap 1991), reduced/selective integration is used in order to integrate the strain energy to overcome this difficulty and is illustrated with a 27 -node hexahedral brick element.

Amongst the more popular formulations are the ones in which compatible displacements and equilibriating stresses are independently interpolated. Stress parameters are eliminated at the element level and a stiffness matrix is obtained, as by Pian (1973). These formulations are called hybrid/mixed formulations. Many three-dimensional hybrid/mixed stress elements have been developed (Zienkiewicz \& Taylor 1989). Here also extravariational techniques like reduced integration and bubble modes are used. Other elements formulated using these principles are 8-node elements (Irons 1972; Lee 1974), 20-node elements (Ahmad \& Irons 1974), special purpose three-dimensional 
elements for thick plate analysis (Spilker 1981) and 20-node quadratic displacement 3-dimensional isoparametric hybrid elements (Spilker \& Singh 1982).

Using this method in which displacement variables are separated, Pian and coworkers were able to formulate isoparametric elements which are coordinate invariant. These elements are known to have certain drawbacks. One of them is the presence of zero energy modes which appear when stress interpolants are used. Pian \& Chen (1983) were able to establish a method which prevented the appearance of zero energy deformation modes. This concept was extended by Pian \& Tong (1986) to develop hexahedral hybrid elements. Spilker \& Singh (1982) developed a 20-node hybrid hexahedral element based on the complementary energy principle. As the stress interpolants need to satisfy equilibrium conditions, an a priori cartesian coordinate interpolation was necessary. Such interpolation is known to provide poor stresses and displacements under grossly distorted conditions. Another such element was formulated by Loikkanen \& Irons (1984). Punch \& Atluri (1984a, 1984b) used symmetric group theory to study a number of such elements (using cartesian interpolation functions) for stability, coordinate invariance and for the optimal stress functions to be chosen. Tang \& Chen (1982) proposed a series of non-conforming stress based elements. Even though their performance was better than those of conforming ones, they were no superior to the modified Wilson 8-node brick. Chen \& Cheung (1987) derived a new functional (as a generalization of the functional proposed by Pian in 1964, with displacements, stresses and strains as independent variables) to obtain a series of isoparametric elements (Cheung \& Chen 1988). However, the formulation of these elements requires an additional stress field and proper matching of displacements, strains and stresses. This requires experience.

Bachrach (1987) used a $\gamma$-projection operator in tandem with the $\mathrm{Hu}$-Washizu principle to obtain a stress-based element which performed well in bending and near incompressibility. The element required to be reduced integrated and stresses extrapolated to the nodes. Yunus et al (1989) have formulated a hybrid solid element with rotational degrees of freedom. Sze et al (1990) by using a modified HellingerReissner principle, with prior constraints and recognition of the orthogonality of strains, stresses and incompatible displacements obtain a series of isoparametric elements. These could be reduced integrated. Chen \& Cheung (1992) by using a weaker constraint condition on their previous formulation (Cheung \& Chen 1988) were able to obtain elements with improved performance. Dong \& de Freitas (1992) obtained an isoparametric incompatible solid element based on the Hellinger-Reissner principle which was modified by the presence of a constant stress multiplier. Sze \& Ghali (1993) start with the assumed stress element of Pian \& Tong (1986), identify the strain components which cause locking and selectively scale them down to obtain an incompatible element. The element is then corrected to pass the patch test using an "admissible matrix formulation". Andelfinger \& Ramm (1993) use the enhanced assumed strain method (EAS) (obtained by the use of an extra strain field - not continuous) to obtain an 8-node element. Pian (1985) by a modification of his original procedure, by choosing the displacement field in a "consistent" manner, was able to obtain a hybrid 8-node solid element with improved performance under distorted conditions. The growth in popularity and reliability of current day symbolic manipulators has speeded up the development of solid elements. Tan et al (1991) have elaborated on procedures to formulate an 8-node hybrid hexahedral element.

All the elements mentioned above have been compared individually against the displacement based solid elements. Comparisons are normally made against standard 
problems proposed in literature. (MacNeal \& Harder 1985; Belytschko \& Liu 1986; White \& Abel 1989). Kim et al (1990) have compared the performance of the degenerated 20-node element against the 18-node assumed strain element.

All the solid elements discussed do not yet seem to show completely correct predictions for stresses as well as displacements encompassing different types of loading conditions.

\section{Elements based on solutions to the Navier equation}

All the elements discussed in the preceding sections seem to be formulated with the express desire of obtaining good element performance. Instead of attacking the root of the problem, a precise statement of which does not seem to exist so far, they seem to provide ways and means of getting around it. Even methods like the field consistency concept and the use of physical shape functions, which attempt to get to the bottom of the problem, do not as yet appear to be perfected for solid finite elements.

We have looked at the problem afresh. Recognizing the fact that the overall element behaviour seems to be guided by the interpolation functions chosen, we have formulated two hexahedral elements (Venkatesh \& Shrinivasa 1993a) using Papcovitch-Neuber solutions as interpolation functions which satisfy the governing differential equations (Navier equations) a priori (Venkatesh \& Shrinivasa 1993b). It was felt that such a method would combine the simplicity of the displacement-based approach (the Navier equations are written in terms of displacements) and accurate stress prediction of the stress-based approach (the Navier equations are based on equations of stress equilibrium). Such a method has been thought of before (Hoppe 1973), but does not seem to have been implemented for solid elements.

These elements (Venkatesh \& Shrinivasa 1993c, 1993d) have been formulated without taking recourse to any of the above mentioned extra-variational techniques. The elements are exactly integrated by breaking the hexahedra into five tetrahedra (Stroud 1971) and then using appropriate gauss point integration. They exhibit correct element behaviour when subjected to bending moment loads even under grossly distorted conditions and also when the stresses are computed directly by evaluating them at the nodes instead of "at some optimal locations within the elements" (Barlow 1976, 1989). The element performance for out-of-plane shear matches with that of the Wilson 8-node brick for rectangular elements and has been shown to be better than the latter under distorted conditions. The elements pass the constant strain patch test under rectangular conditions. Under distorted conditions they pass the "weak patch test" (Cook et al 1989). Relative performance of the these elements vis-a-vis already existing ones can be found in Venkatesh \& Shrinivasa (1993e).

It therefore appears that it would be possible to formulate finite elements which do not experience any irrational behaviour by simply using the solutions to the Navier equations as interpolation functions.

\section{Conclusion}

We have reviewed the development of solid finite elements since the inception of the finite element method. We have looked at displacement based elements and their drawbacks such as the presence of shear locking etc. We have briefly reviewed the 
extra-variational techniques used in literature to overcome these drawbacks. The paper also reviews the state-of-the-art lower-order hexahedral finite elements being currently proposed. Finally, we critically discuss the lower-order finite elements developed by using interpolation functions which satisfy the governing differential equations a priori and conclude that it is possible to formulate such finite elements without taking recourse to any extra-variational techniques.

The authors wish to acknowledge the support received for carrying out this work from the Aeronautics Research and Development Board through grant no. AERO/RD$134 / 100 / 10 / 90-91 / 653$.

\section{References}

Ahmad S, Irons B M 1974 An assumed stress approach to refined isoparametric finite elements in three dimensions. In Finite element methods in engineering, University of New South Wales

Ahmad S, Irons B M, Zienkiewicz O C 1970 Analysis of thick and thin shell structures by curved elements. Int. J. Numer. Methods Eng. 2: 419-451

Andelfinger U, Ramm E 1993 EAS-elements for two-dimensional, three-dimensional, plate and shell structures and their equivalence to HR-elements. Int. J. Numer. Methods Eng. 36: 1311-1337

Argyris J H 1965 Matrix analysis of three-dimensional elastic media small and large displacements. AIAA J. 3: 45-51

Argyris J H, Fried I 1968a The LUMINA element for the matrix displacement method. Aeronaut. J. R. Aeronaut. Soc. 72: 514-517

Argyris J H, Fried I, Scharpf D W 1968b The HERMEs8 element for the matrix displacement method. Aeronaut. J. R. Aeronaut. Soc. 72: 613-617

Argyris J H, Fried I, Scharpf D W 1968c The TET20 and TEA8 elements for the matrix displacement method. Aeronaut. J. R. Aeronaut. Soc. 72: 618-623

Bachrach W E 1987 An efficient formulation of hexahedral elements with high accuracy for bending and incompressibility. Comput. Struct. 26: 45-467

Barlow J 1976 Optimal stress locations in finite element models. Int. J. Numer. Methods Eng. 10: $243-251$

Barlow J 1989 More on optimal stress points-reduced integration, element distortions and error estimation. Int. J. Numer. Methods Eng. 28: 1487-1504

Bathe K, Wilson E L 1976 Numerical methods in finite element analysis (Englewood Cliffs, NJ: Prentice-Hall)

Bathe K J, Dvorkin E N 1985 A four-node plate bending element based on the Mindilin/Reissner plate theory and mixed interpolation. Int. J. Numer. Methods Eng. 21: 367-383

Belytschko T, Liu W K 1986 Test problems and anomalies in shell finite elements. In Reliability of methods for engineering analysis Proc. Int. Conf. Univ. College (Swansea: UK: Pineridge Press) pp 393-406

Bergan P G, Nygard M K 1984 Finite elements with increased freedom in choosing shape functions. Int. J. Numer. Methods Eng. 20: 643-663

Brassioulis D 1989 On the basics of the shear locking problem of $C^{0}$ isoparametric plate elements. Comput. Struct. 33: 169-185

Bretl J L, Cook R D 1979 A new eight-node solid element. Int. J. Numer. Methods Eng. 14: 593-615

Case W R, Mitchell R S, Vandegrift R E 1986 Improved accuracy in solid isoparametric elements using selectively reduced integration. NASA Conference Publication 2373, NASA, Washington, DC, p. 31

Case W R, Vandegrift R E 1986 Improved isoparametric solid and membrane elements. NASA Conference Publication 2419, NASA, Washington, DC, pp. 28-55 
Chacour S 1972 'DANUTA', a three-dimensional finite element program used in the analysis of turbomachinery. J. Basic Eng., ASME 94: 71-77

Chandra S, Prathap G 1989 A field-consistent formulation for the 8-noded solid finite element. Comput. Struct. 33: 345-355

Chen W, Cheung Y K 1987 A new approach for the hybrid element method. Int. J. Numer. Methods Eng. 24: 1697-1709

Chen W, Cheung Y K 1992 Three-dimensional 8-node and 20-node refined hybrid isoparametric elements. Int. J. Numer. Methods Eng. 35: 1871-1889

Cheung Y K, Chen W 1988 Isoparametric hybrid hexahedral elements for three dimensional stress analysis. Int. J. Numer. Methods Eng. 26: 677-693

Ciarlet P G 1978 The finite element method for elliptic problems (New York: North Holland)

Clough R W 1969 Comparison of three-dimensional finite elements In Symposium on application of finite element methods in civil engineering (eds) W H Rowan, R M Hackett (Nashville, TN: ASCE)

Collatz L 1950 The numerical treatment of differential equations (Berlin: Springer-Verlag)

Conte S D, de Boor C 1980 An introduction to numerical analysis, an algorithmic approach (Singapore: McGraw Hill)

Cook R D 1977 More about artificial softening of finite elements. Int. J. Numer. Methods Eng. 11: 1334-1339

Cook R D, Malkus D S, Plesha M E 1989 Concepts and applications of finite element analysis (New York: John Wiley and Sons)

Courant R 1943 Variational methods for the solution of problems of equilibriums and vibrations. Bull. Am. Math. Soc. 49: 1-23

Courant R, Hilbert D 1953 Methods of mathematical physics (New York: John Wiley and Sons)

Crisfield M A 1984 A quadratic mindilin element using shear constraints. Comput. Struct. 18: $833-852$

de Arrantes Oliveira E R 1968 Theoretical foundations of the finite element method. Int. J. Solids Struct. 4: 929-952

de Freitas J A T, Castro L M S 1992 Digital interpolation in mixed finite element structural analysis. Comput. Struct. 44: 743-751

Donea J, Lamain L G 1987 A modified representation of transverse shear $C^{0}$ quadrilateral plate elements. Comput. Methods. Appl. Mech. Eng. 63: 183-207

Dong Y F, de Freitas J A T 1992 An efficient 8-node incompatible solid element with stress interpolation. Comput. Struct. 44: 773-781

Ergatoudis J, Irons B M, Zienkiewicz O C 1968 Three-dimensional analysis of arch dams and their foundations. In Symposium on arch dams (London: Institution of Civil Engineers)

Ferguson G H, Clark R D 1979 A variable thickness, curved beam and shell stiffening element with shear deformations. Int. J. Numer. Methods. Eng. 14: 581-592

Fjeld S A three-dimensional theory of elasticity. In Finite element methods in stress analysis (eds) I Holland, K Bell (Trondheim: Tapir Norway)

Flaganan D P, Belytschko T 1981 A uniform strain hexahedron and quadrilateral with orthogonal hour glass control. Int. J. Numer. Methods. Eng. 17: 679-706

Fried I 1974 Residual energy balancing technique in the generation of plate bending elements. Comput. Struct. 8: 771-778

Fried I 1975 Finite element analysis of thin elastic shells with residual energy balancing and the role of rigid body modes. J. Appl. Mech. 42: 99-104

Gallagher R H, Padlog J, Bijlard P P 1962 Stress analysis of heated complex shapes. J. Am. Rocket Soc. 32: 700-707

Gilewski W, Gomulinski A 1990 Physical shape functions: a new concept in finite elements. Finite Element News (3): 20-23

Haggenmacher G W 1993 The shape of things. III. Finite Element News (2): 38-41

Heppler G R, Hansen J S 1987 Timoshenko beam finite elements using trigonometric basis functions. $A I A A$ J. 26: 1378-1386

Hoppe V 1973 Finite elements with harmonic interpolation functions. In The mathematics of finite elements and applications (ed.) J R Whiteman (New York: Academic Press)

Hughes J R, Allik H 1969 Finite elements for compressible and incompressible continua. In Symposium on application of finite element methods in civil engineering (ed.) W H Rowan, R M Hackett (Nashville, TN: ASCE) 
Irons B M 1966 Engineering applications of numerical integration in stiffness methods. $A I A A$ J. 4: $2035-2037$

Irons B M 1972 An assumed stress version of the Wilson 8-node brick. University of Wales, Computer Report CNME/CR/56

Irons B M, Ahmad S 1979 Techniques of finite elements (Chichester: Ellis Horwood)

Kelen P 1989 Control of spurious nodes in 20-noded isoparametric element. Commun. Appl. Numer. Methods 5: 415-422

Kidger D J 1990 The 14-node brick element family. Finite Elements Anal. Design 6:

Kim Y H, Jones R F, Lee S W 1990 Study of 20-node solid element. Commun. Appl. Numer. Methods 6: 197-205

Lee S W 1974 An assumed stress hybrid finite element for three-dimensional elastic structural analysis. Massachusetts Institute of Technology, ASRL-TR-170-3

Loikkanen M J, Irons B M 1984 An 8-node brick finite element. Int. J. Numer. Methods. Eng. 20: $523-528$

MacNeal R H 1982 Derivation of assumed strain matrices by assumed strain distributions. Nucl. Eng. Design 70: 3-12

MacNeal R H, Harder R L 1985 A proposed set of problems to test finite element accuracy. Finite Elements Anal. Design 1: 3-20

MacNeal R H 1992 On the limits of finite element perfectibility. Int. J. Numer. Methods. Eng. 35: $1589-1601$

Melosh R J 1963 Structural analysis of solids. J. Struct. Div. ASCE 89: 205-223

Naganarayana B P 1991 Consistency and correctness principles in quadratic displacement type finite elements. Ph D thesis, Dept. Aerospace Eng., Indian Institute of Science, Bangalore

Naganarayana B P, Prathap G 1991 Field-consistency analysis of 27-noded hexahedral elements for constrained media elasticity. Finite Elements Anal. Design 9: 149-168

Nguyen V P 1982 Automatic mesh generation with tetrahedron elements. Int. J. Numer. Methods. Eng. 18: 273-289

Oden J T 1983 Finite elements - mathematical aspects (Englewood Cliffs, NJ: Prentice-Hall)

Oleson J F 1983 Field redistribution in finite elements - a mathematical alternative to reduced integration. Comput. Struct. 17: 157-159

Pammer Z, Szabo L 1981 Stereo decomposition subroutines for three-dimensional plotter programs. Int. J. Numer. Methods. Eng. 17: 1571-1575

Park K C 1984 Symbolic fourier analysis procedures for $C^{0}$ finite elements. In Innovative Methods Nonlinear Anal. (Swansea, UK: Pineridge Press) 269-293

Pawlak T P, Yunus S M, Cook R D 1991 Solid elements with rotational degrees of freedom. Part II. Tetrahedron elements. Int. J. Numer. Methods. Eng. 31: 593-610

Pawsey S F, Clough R W 1971 Improved numerical integration of thick shell finite elements. Int. J. Numer. Methods. Eng. 3: 575-586

Peano A G 1976 Hierarchics of conforming finite elements for elasticity and plate bending. Comput. Math. Appl. 2: 3-4

Pian T H H 1973 Hybrid models. In Numerical methods and computer methods in applied mechanics (eds) S J Fenves, N Perrone, A R Robinson, W C Schnobrich (New York: Academic Press)

Pian T H H 1985 Finite elements based on consistently assumed stresses and displacements. Finite Elements Anal. Design 1: 131-140

Pian T H H, Chen D P 1983 On the suppression of zero energy deformation modes. Int. J. Numer. Methods. Eng. 19: 1741-1752

Pian T H H, Tong P 1986 Relations between incompatible displacement model and hybrid stress model. Int. J. Numer. Methods. Eng. 22: 173-181

Prathap G 1985 A $C^{0}$ continuous 4-noded cylindrical shell element. Comput. Struct. 21: 995-999

Prathap G 1992 Recent advances in finite element technology. NAL-UNI Series, National Aerospace Lab., Bangalore

Punch E F, Atluri S N 1984a Applications of isoparametric three-dimensional hybrid-stress finite elements with least-order fields. Comput. Struct. 19: 406-430

Punch E F, Atluri S N 1984b Development and testing of 2- and 3-d hybrid stress elements. Comput. Methods. Appl. Mech. Eng. 47: 331-356

Rashid Y R 1969; 1970 Three dimensional analysis of elastic solids: I. Analysis procedure; II. The computational problems. Int. J. Solids Struct. 5: 6: 1311-1331; 195-207 
Rashid Y R, Smith P D, Prince N 1969 On further application of finite element method to three-dimensional elastic analysis. In Symposium on high speed computing of elastic structures (eds) W H Rowan, R M Hackett (Nashville, TN: ASCE)

Rigby G L, McNeice G M 1972 A strain energy basis for studies of element stiffness matrices. AIAA J. 10: 1490-1493

Robinson J 1985 Early FEM pioneers (Dorset: Robinson and Associates)

Schulz J C 1985 Finite element hourglassing control. Int. J. Numer. Methods. Eng. 21: 1039-1048

Smith I M, Kidger D J 1992 Elastoplastic analysis using the 14-node brick element family. Int. J. Numer. Methods. Eng. 1263-1275

Spilker R L 1981 High-order three-dimensional hybrid-stress elements for thick plate analysis. Int. J. Numer. Methods. Eng. 17: 53-69

Spilker R L, Singh S P 1982 Three-dimensional hybrid-stress isoparametric quadratic displacement elements. Int. J. Numer. Methods. Eng. 18: 445-465

Stolarski H, Belytschko T 1981 Membrane locking and reduced integration for curved beam elements. J. Appl. Mech. 49: 172-178

Strang G 1972 Variational crimes in the finite element method. In The mathematical foundations of the finite element method with applications to partial differential equations (ed.) A K Aziz (New York: Academic Press)

Strang G, Fix G J 1973 An analysis of the finite element method (Englewood Cliffs, NJ: Prentice-Hall)

Stroud A H 1971 Approximate calculation of multiple integrals (Englewood-Cliffs, NJ: Prentice-Hall)

Synge J L 1957 The hypercircle in mathematical physics (London: Cambridge University Press)

Sze K Y, Chow C L, Chen W 1990 A rational formulation of isoparametric hybrid stress elements. Finite Elements Anal. Design 7: 61-72

Sze K Y, Ghali A 1993 Hybrid hexahedral element for solids, plates, shells and beams by selective scaling. Int. J. Numer. Methods. Eng. 36: 1519-1540

Tan H Q, Chang T Y P, Zheng D 1991 On symbolic manipulation and code generation of a hybrid three-dimensional solid element. Eng. Comput. 7: 47-59

Tang L, Chen W 1982 The non-conforming finite elements for stress analysis. In Proceedings in the International Conference on finite element methods (eds) G He, Y K Cheung (New York: Gordon and Breach)

Taylor R L, Beresford P J, Wilson E L 1976 A non-conforming element for stress analysis. Int. J. Numer. Methods. Eng. 10: 1211-1219

Tessler A, Dong S B 1981 On a hierarchy of conforming beam elements. Comput. Struct. 14: 335-344

Turner M J, Clough R W, Martin H C, Topp L J 1956 Stiffness and deflection analysis of complex structures. J. Aeronaut. Sci. 23: 805-823

Venkatesh D N, Shrinivasa U 1993a Formulation of finite elements which satisfy the Navier equation a priori. Report 93 VAR5. Dept. Mech. Eng. Indian Institute of Science, Bangalore

Venkatesh D N, Shrinivasa U 1993b Solutions to the Navier equation and equivalent Papcovitch-Neuber functions. Report 93 var 1, Dept. Mech. Eng. Indian Institute of Science, Bangalore

Venkatesh D N, Shrinivasa U 1993c Generation of an 8-node brick element using PN functions. Report 93 VAR2, Dept. Mech. Eng. Indian Institute of Science, Bangalore

Venkatesh D N, Shrinivasa U 1993d Hexahedral elements using PN functions for beam problems. Report 93 VAR3, Dept. Mech. Eng. Indian Institute of Science, Bangalore

Venkatesh D N, Shrinivasa U 1993e Performance study of hexahedral elements using PN functions. Report 93 var5, Dept. Mech. Eng. Indian Institute of Science, Bangalore

Vlachoutsis S 1990 Explicit integration of three-dimensional degenerated shell elements. Int. J. Numer. Methods. Eng. 29: 861-880

Wachspress E I 1975 Rational finite element basis (New York: Academic Press)

Wait R 1971 A finite element for 3-dimensional function approximation. Proc. Conf. Appl. Numer. Anal. Springer-Verlag lecture notes in Mathematics (Berlin, Heidelberg: SpringerVerlag) 228: $348-352$

Washizu K 1968 Variational principles in elasticity and plasticity (London: Pergamon)

White D W, Abel J F 1989 Testing of shell finite element accuracy and robustness. Finite Elements Anal. Design 6: 129-151 
Wilson E L 1973 Incompatible displacement models, In Numerical methods and computer methods in applied mechanics (eds) S J Fenves, N Perrone, A R Robinson, W C Schnobrich (New York: Academic Press)

Wilson E L, Ibrahimbegovic A 1990 Use of incompatible displacement modes for the calculation of element stiffnesses or stresses. Finite Elements Anal. Design. 7: 229-241

Wilson E L, Taylor R L, Doherty W P, Ghaboussi T 1973 Incompatible displacement models. In Numerical Computational Methods in Structural Mechanics (eds) S J Fenves et al (New York: Academic Press)

Yang T Y 1986 Finite element structural analysis (Englewood Cliffs, NJ: Prentice-Hall)

Yu H S 1990 A rational displacement interpolation function for axisymmetric finite element analysis of nearly incompressible materials. Finite Elements Anal. Design 10: 205-219

Yunus S M, Pawlak T P, Cook R D 1991 Solid elements with rotational degrees of freedom: Part I. Hexahedron elements. Int. J. Numer. Methods. Eng. 31: 573-592

Yunus S M, Saigal S, Cook R D 1989 On improved hybrid elements with rotational degrees of freedom. Int. J. Numer. Methods. Eng. 28: 785-800

Zienkiewicz O C 1973 Finite elements - the background story. In The mathematics of finite elements and applications (ed.) J R Whiteman (New York: Academic Press)

Zienkiewicz O C 1977 The finite element method (New York: McGraw Hill)

Zienkiewicz O C, De J P, Gago S R, Kelly D W 1983 The hierarchical concept in finite element analysis. Comput Struct. 16: 53-65

Zienkiewicz O C, Irons B M, Ergatoudis J, Ahmad S, Scott F C 1969 Isoparametric and associated element families for two and three dimensional analysis. In Finite element methods in stress analysis (eds) I Holland, K Bell (Trondheim, Norway: Tapir)

Zienkiewicz O C, Irons B M, Scott F C, Campbell J S 1970 Three dimensional stress analysis. In Symposium on high speed computing of elastic structures 1: 413-432

Zienkiewicz O C, Taylor R L 1989 The finite element method. Vol. I: Basic formulation and linear problems (New York: McGraw Hill)

Zienkiewicz O C, Taylor R L, Too J M 1971 Reduced integration technique in general analysis of plates and shells. Int. J. Numer. Methods. Eng. 3: 275-290 